\title{
Space Time Codes in Keyhole Channels: Analysis and Design
}

\author{
Shahab Sanayei, Member, IEEE, Ahmadreza Hedayat, Member, IEEE, \\ and Aria Nosratinia, Senior Member, IEEE
}

\begin{abstract}
The keyhole condition, where the MIMO channel has only one degree of freedom, impairs the performance of MIMO systems. Thus, one may wish to design codes that are robust to this condition. So far, a general analysis of spacetime codes in keyhole conditions has not been available (except in the special case of orthogonal space-time block codes). This work provides pairwise error probabilities for general space-time codes in keyhole condition. We present design criteria in high SNR, providing guidelines for codes that are robust to keyhole conditions. Also included is the proof of the intuitive result that the diversity under keyhole condition is $\min (M, N)$, where $M$ and $N$ are the number of transmit and receive antennas, with a slightly unexpected twist in the case of $M=N$.
\end{abstract}

Index Terms-Keyhole channels, MIMO, space-time codes.

\section{INTRODUCTION}

I $\mathrm{T}$ has been demonstrated recently [1], [2] that multipleinput multiple-output (MIMO) fading channels can experience a condition known as keyhole or pinhole condition, where despite rich local scattering and uncorrelated transmit and receive signals, the system has only one degree of freedom (Fig. 1). This condition can arise in a number of ways, for example, when there are two rings of scatterers at large separation. Another example is in a hallway or tunnel. Under such conditions, space-time coding will have diminished performance.

Analysis and design of codes under this condition has not been studied extensively. Shin and Lee [3] studied the effect of keyholes on the performance of space-time block codes. Nasri et al. [4] produce one specific example of a space-time trellis code that performs well in the keyhole channel, but they do not present any methodology or design criteria.

In this work, we undertake a general analysis of space-time codes in keyhole channels. The special case of this analysis for orthogonal space-time block codes [3] was made tractable by the helpful structure of these codes that creates an equivalent single-input single-output (SISO) channel. We now solve the general problem in the absence of a simplifying condition.

We present design guidelines for the keyhole condition. Denoting the number of transmit antennas with $M$ and receive antennas with $N$, we report that for $M \leq N$, the design

Manuscript received October 11, 2005; revised May 12, 2006 and August 3, 2006; accepted August 3, 2006. The associate editor coordinating the review of this letter and approving it for publication was H. Jafarkhani. This work was presented in part in Globecom 2004.

S. Sanayei is with ArrayComm, 2480 N. First Street, Suite 200, San Jose, CA 95131-1014 USA (e-mail: sanayei@ieee.org).

A. Hedayat is with Navini Networks, 2240 Campbell Creek Boulevard, Richardson, TX 75082 USA (e-mail: hedayat@ieee.org).

A. Nosratinia is with the Department of Electrical Engineering, The University of Texas at Dallas, Richardson, TX 75080 USA (e-mail: aria@utdallas.edu).

Digital Object Identifier 10.1109/TWC.2007.05782.

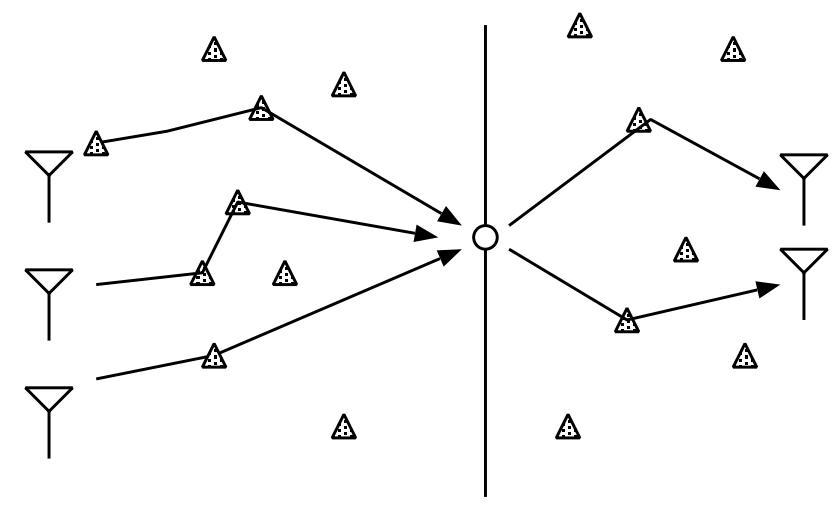

Fig. 1. Keyhole channel.

criteria are similar to non-keyhole condition, but for $M>N$, the design criteria are different.

We also prove a result mentioned in [2], that the diversity order of the keyhole channel is $\min (M, N)$. Although this result is intuitive, to our knowledge no proof of it exists in prior literature. The results presented in the sequel appeared in part in [5].

The notation in this paper is as follows: $\mathbb{E}[]$ refers to expected value of a random variable, $\gamma \approx 0.57721566$ is the Euler-Mascheroni constant and $e$ is the base of natural logarithm. Gamma function is defined as: $\Gamma(x)=\int_{0}^{\infty} t^{x-1} e^{-t} d t$. We use $a_{n} \stackrel{\circ}{=} b_{n}$ to denote the asymptotic equivalence of $a_{n}$ and $b_{n}$ defined as: $\lim _{n \rightarrow \infty} \frac{a_{n}}{b_{n}}=1$.

\section{System Model}

We assume a frequency non-selective linear time invariant fading channel. The signal model is

$$
\mathbf{Y}=\sqrt{\frac{\rho}{M}} \mathbf{H} \mathbf{X}+\mathbf{N}
$$

where $\mathbf{Y}$ is an $N \times T$ matrix representing the received signal, $\mathbf{X}$ is the $M \times T$ transmitted codeword matrix, $\rho$ is the average signal-to-noise ratio (SNR), and $\mathbf{N}$ is the zero mean additive circularly symmetric complex Gaussian noise matrix with size $N \times T$, whose elements have unit variance per dimension. The $N \times M$ MIMO channel matrix is denoted by $\mathbf{H}$ whose $i, j$ element is the channel coefficient between the $j$ transmit antenna and $i$ receive antenna. The channel $\mathbf{H}$ is a keyhole channel modeled as

$$
\mathbf{H}=\mathbf{h} \mathbf{g}^{T}
$$

where $\mathbf{h}$ is a $M \times 1$ vector and $\mathbf{g}$ is an $N \times 1$ vector both with zero mean complex Gaussian entries. In decoding the received codeword $\mathrm{Y}$ we employ maximum likelihood (ML) decoder, 
assuming the channel state information (CSI) is perfectly known at the receiver and unknown at the transmitter.

\section{PAIRWISE ERror Probability}

Approximating FER and BER is usually through pairwise error probability leading to a union bound over the probability of error.

$$
P_{e} \leq \sum_{\mathbf{c} \in \mathcal{C}} P(\mathbf{c}) \sum_{\mathbf{c}^{\prime} \neq \mathbf{c}} P\left(\mathbf{c} \rightarrow \mathbf{c}^{\prime}\right)
$$

where $P\left(\mathbf{c} \rightarrow \mathbf{c}^{\prime}\right)$ is the pairwise error probability (PEP). We derive expressions for the PEP and use PEP expressions to calculate bounds for the probability of error of space time codes. These bounds provide insight into designing better codes under keyhole condition. We use the popular techniques of Simon and Alouini [6], using the moment generating function (MGF). For a given channel realization $H$ the PEP of a Gaussian channel can be calculated as:

$$
P\left(\mathbf{c} \rightarrow \mathbf{c}^{\prime} \mid \mathbf{H}\right)=Q\left(\sqrt{\frac{E_{s}}{2 N_{0}}\|\mathbf{H} \boldsymbol{\Delta}\|^{2}}\right)
$$

where $\boldsymbol{\Delta}=\mathbf{c}-\mathbf{c}^{\prime}$ is the code word difference matrix. It is now well known that using the alternative definition of Q-function by a proper integral [6]

$$
Q(x)=\int_{0}^{\pi / 2} \exp \left(-\frac{x^{2}}{2 \sin ^{2} \theta}\right) d \theta
$$

and integrating over all channel realizations results in a PEP expression involving the MGF, i.e.,

$$
P\left(\mathbf{c} \rightarrow \mathbf{c}^{\prime}\right)=\int_{0}^{\pi / 2} \psi\left(-\frac{E_{s}}{4 N_{0} \sin ^{2} \theta}\right) d \theta
$$

where $\psi($.$) is the MGF of the random variable \|\mathbf{H} \boldsymbol{\Delta}\|^{2}$.

\section{A. Calculation of MGF for the Keyhole Channel}

Under the keyhole condition:

$$
\begin{aligned}
\|\mathbf{H} \boldsymbol{\Delta}\|^{2} & =\left\|\mathbf{h} \mathbf{g}^{T} \boldsymbol{\Delta}\right\|^{2} \\
& =\operatorname{tr}\left(\mathbf{h} \mathbf{g}^{T} \boldsymbol{\Delta} \boldsymbol{\Delta}^{T} \mathbf{g h}^{T}\right)=\operatorname{tr}\left(\mathbf{h}^{T} \mathbf{h} \mathbf{g}^{T} \boldsymbol{\Delta} \boldsymbol{\Delta}^{T} \mathbf{g}\right) \\
& =\|\mathbf{h}\|^{2}\left\|\mathbf{g}^{T} \boldsymbol{\Delta}\right\|^{2}
\end{aligned}
$$

Since $\mathbf{h}$ is a Gaussian vector with jointly independent elements, the random variable $X \triangleq\|\mathbf{h}\|^{2}$ is distributed as $\chi_{2 N}^{2}$. Let $\boldsymbol{\Delta} \boldsymbol{\Delta}^{T}=\mathbf{U}^{T} \mathbf{D U}$ be the eigen decomposition of $\mathbf{A}=\boldsymbol{\Delta} \boldsymbol{\Delta}^{T}$ in which $\mathbf{U}$ is a unitary matrix and $\mathbf{D}=$ $\operatorname{diag}\left(d_{1}, \ldots, d_{M}\right)$ where $d_{1}, \ldots, d_{M}$ are the eigenvalues of $\mathbf{A}$ (since $\mathbf{A}$ is symmetric positive definite, $d_{i}$ are non-negative). The vector $\mathbf{g}$ is circularly symmetric Gaussian vector whose elements are jointly independent, thus $\hat{\mathbf{g}}=\mathbf{U g}$ has the same distribution and we have

$$
Y \triangleq\left\|\mathbf{g}^{T} \boldsymbol{\Delta}\right\|^{2}=\sum_{i=1}^{M} d_{i}\left|\hat{g}_{i}\right|^{2}
$$

Each of the random variables $\left|\hat{\mathrm{g}}_{i}\right|^{2}$ is exponentially distributed, so random variable $\mathbf{Y}$ is non-central chi-square, thus the moment generating of $\mathbf{Y}$ can be calculated as follows:

$$
\psi_{Y}(s)=\mathbb{E}\left[e^{s Y}\right]=\prod_{i=1}^{M} \frac{1}{1-d_{i} s}
$$

We need to calculate the MGF of the product of two random variables, which can be done according to the following lemma.

Lemma 1: Let $Z=X . Y$ where $X$ and $Y$ are two independent positive random variables with pdf $f_{X}($.$) and f_{Y}($.$) and$ let $\psi_{Y}(s)=\mathbb{E}\left[e^{s Y}\right]$ be the MGF of $Y$, Then the MGF of $Z$ can be calculated as follows:

$$
\psi_{Z}(s)=\int_{0}^{\infty} f_{X}(t) \psi_{Y}(s t) d t
$$

Proof: See the appendix.

By direct application of Lemma 1 to the random variable $Z \triangleq$ $\|\mathbf{H} \boldsymbol{\Delta}\|^{2}=X Y$ we obtain the following formula for the MGF of $Z$ :

$$
\psi_{Z}(-s)=\frac{1}{\Gamma(N)} \int_{0}^{\infty} \frac{t^{N-1} e^{-t}}{\prod_{i=1}^{M}\left(1+d_{i} s t\right)} d t
$$

Thus $\left.\psi_{Z}(-s)\right|_{s=\frac{E_{s}}{4 N_{0}}}$ serves an upper bound for PEP in Eq. (5).

\section{B. Asymptotic Behavior of MGF and Diversity Order}

We now look at the behavior of the MGF obtained in Eq. 9 in the asymptote of large $s$. This corresponds to the behavior of the PEP in the asymptote of high SNR.

Theorem 1: Assuming that all $d_{i}$ are strictly positive and distinct, ${ }^{1}$ the behavior of $\psi_{Z}(-s)$ in the asymptote of large $s$ is:

$$
\psi_{Z}(-s) \stackrel{\circ}{=} \begin{cases}\frac{\Gamma(N-M)}{\Gamma(N) \prod_{i=1}^{M} d_{i}} \frac{1}{s^{M}} & N>M \\ \frac{1}{\Gamma(N) \prod_{i=1}^{M} d_{i}} \frac{\log s}{s^{M}} & N=M \\ \left(\frac{(-1)^{N-1}}{\Gamma(N)} \sum_{i=1}^{M} \frac{\log d_{i}}{d_{i}^{N}} \alpha_{i}\right) \frac{1}{s^{N}} & N<M\end{cases}
$$

where $\alpha_{i} \triangleq \prod_{j \neq i} \frac{d_{i}}{d_{i}-d_{j}}$.

Proof: See the appendix.

It is reported in [2] that the diversity order of a keyhole channel is $\min (M, N)$. This is expected because the keyhole channel can be thought of as the cascade of a MISO (with diversity order of $M$ ) and a SIMO (with diversity order of $N$ ). Theorem 1 verifies this result. In the MISO-SIMO decomposition, the channel with the smaller diversity is the bottleneck for data transmission, hence the diversity order of the keyhole channel cannot be more than $\min (M, N)$. Theorem 1 also shows that when $M=N$, the MGF has the form $\log (s) / s^{M}$, which dominates $1 / s^{M-1}$ but is dominated by $1 / s^{M}$. Finally, Theorem 1 provides the coding gain, which can be used for designing better codes for the keyhole channel.

\section{Code Design Criterion}

For the case $N \geq M$, Theorem 1 says that the determinant criterion from [7] also applies to the keyhole channel, thus the codes reported for the Rayleigh fading channel will also

${ }^{1}$ When $d_{i}$ are not distinct a similar result holds. For example, when $d_{1}=$ $. .=d_{M}=d$ then the behavior of $\psi_{Z}(-s)$ in the asymptote of large $s$ is:

$$
\psi_{Z}(-s) \stackrel{\circ}{=} \begin{array}{ll}
\frac{\Gamma(N-M)}{\Gamma(N) d^{M}} \frac{1}{s^{M}} & N>M \\
\frac{1}{\Gamma(N) d^{M} \frac{\log s}{s^{M}}} & N=M \\
\frac{1}{\Gamma(N)(M-N+1) d^{N}} \frac{1}{s^{N}} & N<M
\end{array}
$$




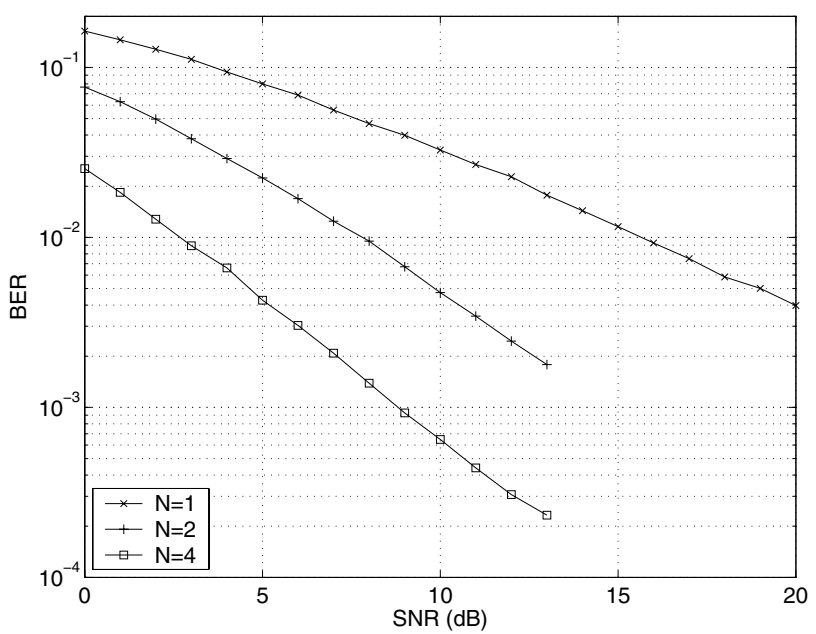

Fig. 2. Bit error rate of the four-state QPSK STT code in keyhole channel.

perform well under keyhole conditions if $N \geq M$. However, if $N<M$, the coding gain will have a new expression, and the space-time code design criterion must be based on a metric different from that in [7]. For example, in the simple case of $M=2$ and $N=1$, the bound on PEP reduces to:

$$
P E P \leq\left(\frac{\log d_{1}-\log d_{2}}{d_{1}-d_{2}}\right) \frac{E_{s}}{4 N_{0}}
$$

which is the same result reported in [4]. In the general case, the code design criterion is based on minimizing the following expression:

$$
(-1)^{N-1} \sum_{i=1}^{M} \frac{\log d_{i}}{d_{i}^{N}}\left(\prod_{j \neq i} \frac{d_{i}}{d_{i}-d_{j}}\right) .
$$

\section{Application And Results}

In this section we apply the new criterion to design improved codes in two categories: space-time trellis codes and super-orthogonal codes. In the plots, the limit-beforeaveraging technique of Malkamäki and Leib [8] has been used to generate tighter bounds.

In the first example, we employ the four-state QPSK STT code of [7]. This code is designed for $M=2$ and achieves the transmit diversity of two in i.i.d. MIMO channel. As stated earlier, in keyhole channels, the diversity of a spacetime signaling is determined by $\min (M, N)$. Hence, for this code, we expect the diversity of one when $N=1$ and diversity of two when $N \geq 2$. Fig. 2 and Fig. 3 show the bit error rate (BER) and frame error rate (FER) of the code with $N=1,2,4$ in keyhole channel. We observe that for $N=1$ the diversity of the code is one. The code shows the diversity of two for $N=4$. For $N=2$, the slope of the error curve is slightly less than two which is explained by the $\log s$ term in the MGF of Theorem 1 when $N=M$. The union bound of the FER for $N=1,2$ are also shown in Fig. 3.

The design rule of (10) and (11) suggests a different coding gain design criterion compared to that of [7] when $M>N$. We would like to see if the above STT code can be redesigned to achieve better performance in keyhole channels. The trellis

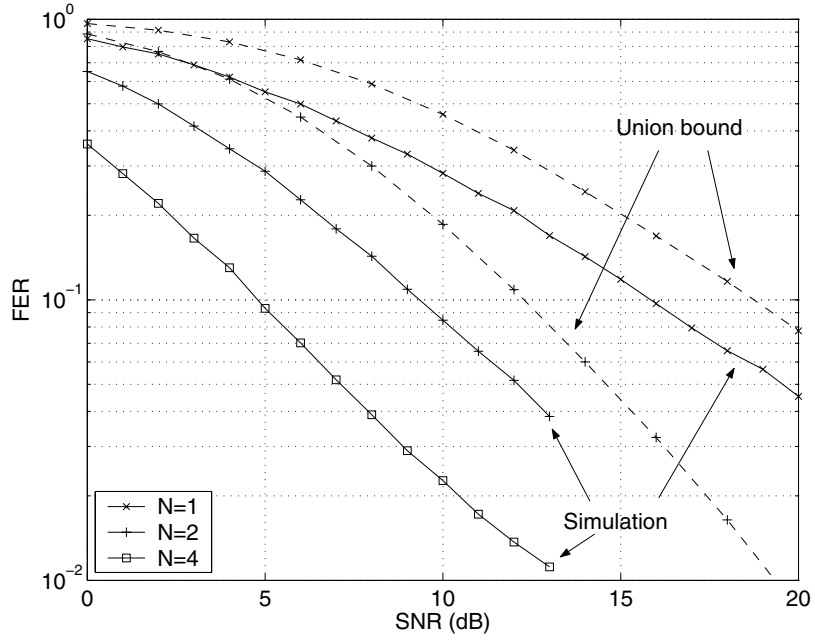

Fig. 3. Frame error rate of the four-state QPSK STT code in keyhole channel.

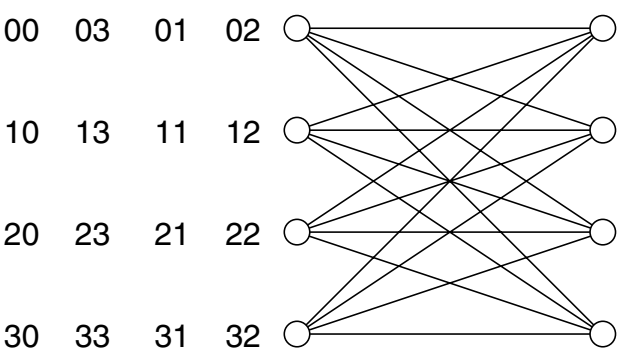

Fig. 4. A Space-Time trellis code with improved performance in keyhole channel.

of the modified code is shown in Fig. 4. This code was obtained by permuting the trellis of [7] such that the coding gain according to the new criterion is increased. In principle this can be done through exhaustive search.

The performance of the new code and the original code of [7], in both keyhole channel and i.i.d. MIMO channel with $N=1,2$, are shown in Fig. 5 . We observe that the new code achieves about $1 \mathrm{~dB}$ gain in keyhole channel with $N=1$ over the code of [7]. The new code gives slightly better performance even in quasi-static i.i.d. channel. The improved performance of the code in the i.i.d. channel and for $N=2$ is due to the minimum determinant criterion of [7], whose multiplicity is smaller in the modified code compared to the original code. We mention that the code of [4] also corresponds to a good value of our metric and therefore produces similar (slightly inferior) results compared to our example.

In the second example, we investigate the so-called superorthogonal codes of Jafarkhani and Seshadri [9]. In particular, we look at two codes in this category: the twostate BPSK super-orthogonal code, and the two-state QPSK super-orthogonal code. In each of these cases, we find that the new design criterion does not instruct us to change the trellis labelings, however, the set partitioning will be changed. Specifically, in the BPSK case, the set partitioning will change from the ordered set $(00,11,01,10)$ to $(00,10,01,11)$, and in 


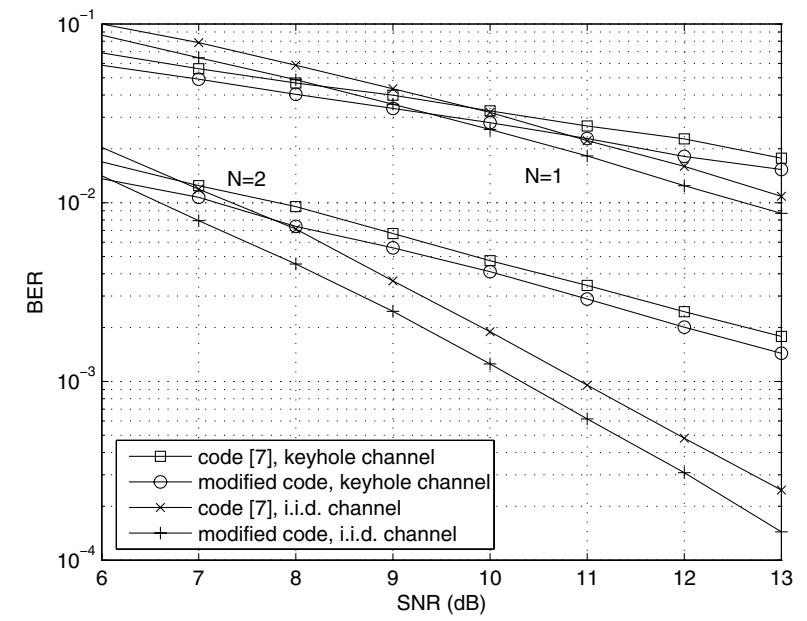

Fig. 5. Bit error rate of the four-state QPSK STT code and the modified code under i.i.d. and keyhole conditions.

the QPSK case, the set partitioning in [9] was

$(00,22,02,20,11,33,13,31,01,23,03,21,10,32,12,30)$, which according to the new criterion is changed to

$(00,01,02,03,10,11,12,13,20,21,22,23,30,31,32,33)$.

The performance of the original as well as modified superorthogonal codes in keyhole channels are demonstrated in Fig. 6.

\section{Discussion AND CONClusion}

This paper presents a general analysis of space-time codes in keyhole channels. Using this analysis, we provide design criteria for codes that are robust to the keyhole condition. We report that for the case of $M \leq N$, the design criteria in the keyhole condition are no different from the criteria under rich scattering, but for $M>N$ the design criteria are indeed affected. We design new codes under the new design criterion. We also prove that the diversity order of a MIMO keyhole channel is equivalent to $\min (N, M)$.

\section{APPENDIX}

Proof: [Lemma 1] We know that the pdf of the product of two independent positive random variable $X$ and $Y$ can be calculated as:

$$
f_{Z}(z)=\int_{0}^{\infty} f_{X}(t) f_{Y}\left(\frac{z}{t}\right) \frac{d t}{t}
$$

hence,

$$
\begin{aligned}
\psi_{Z}(s) & =\mathbb{E}\left[e^{s Z}\right]=\int_{0}^{\infty} e^{s z} f_{Z}(z) d z \\
& =\int_{0}^{\infty} \int_{0}^{\infty} e^{s z} f_{x}(t) f_{Y}\left(\frac{z}{t}\right) \frac{d t}{t} d z \\
& =\int_{0}^{\infty} f_{x}(t) d t \int_{0}^{\infty} e^{s z} f_{Y}\left(\frac{z}{t}\right) \frac{d z}{t}
\end{aligned}
$$

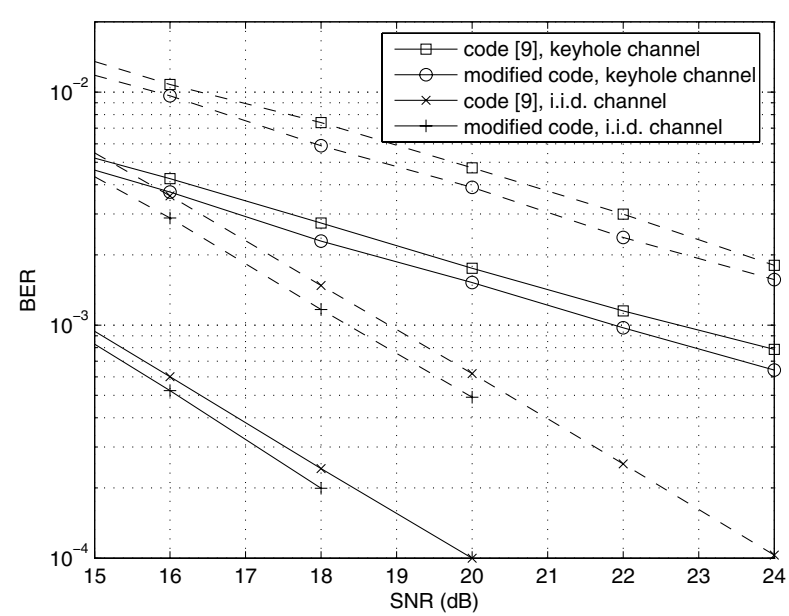

Fig. 6. Original and modified super-orthogonal codes with BPSK (solid lines) and QPSK (dashed lines) under i.i.d. and keyhole conditions.

we make a change of variable $u=\frac{z}{t}$, hence we get:

$$
\begin{aligned}
\psi_{Z}(s) & =\int_{0}^{\infty} f_{X}(t) d t \int_{0}^{\infty} e^{s t u} f_{Y}(u) d u \\
& =\int_{0}^{\infty} f_{x}(t) \psi_{Y}(s t) d t
\end{aligned}
$$

To prove Theorem 1, we first prove the following two lemmas.

\section{Lemma 2: Let}

$$
\alpha_{i}=\prod_{0 \leq j \leq M, j \neq i} \frac{d_{i}}{d_{i}-d_{j}}
$$

given that $d_{i} \neq d_{j}$ for all $i \neq j$ and $d_{i} \neq 0$ for all $i$. We have:

$$
\sum_{i=1}^{M} \frac{\alpha_{i}}{d_{i}^{k}}=\left\{\begin{array}{lc}
1 & k=0 \\
0 & 0 \leq k \leq M-1 \\
\frac{(-1)^{M-1}}{\prod_{i=1}^{M} d_{i}} & k=M .
\end{array}\right.
$$

Proof: Assuming $d_{i}$ are distinct, $\alpha_{i}$ is the residue of the simple pole $s=-1 / d_{i}$ of the complex function $f(s)$, defined below, and we have

$$
f(s) \triangleq \prod_{i=1}^{M} \frac{1}{1+d_{i} s}=\sum_{i=1}^{M} \frac{\alpha_{i}}{1+d_{i} s} .
$$

We notice that $f(s)$ has a zero at $s=\infty$ of multiplicity $M$, thus the Laurent expansion of $f(s)$ starts with $1 / s^{M}$, i.e.

$$
f(s)=\sum_{k=M}^{\infty} \frac{f_{k}}{s^{k}},|s|>\max _{1 \leq i \leq M} d_{i}^{-1} .
$$

On the other hand :

$$
\begin{aligned}
\frac{\alpha_{i}}{1+d_{i} s} & =\sum_{k=1}^{\infty} \frac{(-1)^{k-1} \alpha_{i}}{d_{i}^{k}} \frac{1}{s^{k}},|s|>d_{i}^{-1} \\
\sum_{i=1}^{M} \frac{\alpha_{i}}{1+d_{i} s} & =\sum_{i=1}^{M} \sum_{k=1}^{\infty} \frac{(-1)^{k-1} \alpha_{i}}{d_{i}^{k}} \frac{1}{s^{k}} .
\end{aligned}
$$


Since (17) and (18) are equal we have for all $1 \leq k \leq M-1$ :

$$
\begin{aligned}
\sum_{i=1}^{M} \frac{\alpha_{i}}{d_{i}^{k}} & =0 \\
\sum_{i=1}^{M} \frac{\alpha_{i}}{d_{i}^{M}} & =\frac{(-1)^{M-1}}{\prod_{i=1}^{M} d_{i}} .
\end{aligned}
$$

If in (16) we put $s=0$ we get $\sum \alpha_{i}=1$ and this completes the proof.

Lemma 3: The integral $\int_{0}^{\infty} \frac{t^{N-1}}{1+d_{i} s t} e^{-t} d t$ behaves asymptotically as follows

$$
\begin{aligned}
\int_{0}^{\infty} \frac{t^{N-1}}{1+d_{i} s t} e^{-t} d t & \stackrel{\circ}{=} \sum_{k=1}^{N-1} \frac{(-1)^{k-1} \Gamma(N-K)}{d_{i}^{k} s^{k}} \\
& +\frac{(-1)^{N-1}(\log s+\gamma)}{d_{i}^{N} s^{N}}+\frac{(-1)^{N-1} \log d_{i}}{d_{i}^{N} s^{N}}
\end{aligned}
$$

Proof: Let $I_{k}=\int_{0}^{\infty} \frac{t^{k-1}}{1+d_{i} s t} e^{-t} d t$. We have:

$$
\begin{aligned}
I_{k} & =\int_{0}^{\infty} \frac{t^{k-1}+\frac{t^{k-2}}{d_{i} s}}{1+d_{i} s t} e^{-t} d t-\int_{0}^{\infty} \frac{\frac{t^{k-2}}{d_{i} s}}{1+d_{i} s t} e^{-t} d t \\
& =\frac{1}{d_{i} s} \int_{0}^{\infty} t^{k-2} e^{-t} d t-\frac{1}{d_{i} s} \int_{0}^{\infty} \frac{t^{k-2}}{1+d_{i} s t} e^{-t} d t \\
& =\frac{\Gamma(k-1)}{d_{i} s}-\frac{1}{d_{i} s} I_{k-1} .
\end{aligned}
$$

Multiplying both sides of the last equation by $(-1)^{k} d_{i}^{k} s^{k}$, we get

$$
\begin{array}{r}
(-1)^{k} d_{i}^{k} s^{k} I_{k}=(-1)^{k} d_{i}^{k-1} s^{k-1} \Gamma(k-1) \\
+(-1)^{k-1} d_{i}^{k-1} s^{k-1} I_{k-1} \\
\sum_{k=2}^{N}(-1)^{k} d_{i}^{k} s^{k} I_{k}-(-1)^{k-1} d_{i}^{k-1} s^{k-1} I_{k-1} \\
=\sum_{k=2}^{N}(-1)^{k} d_{i}^{k-1} s^{k-1} \Gamma(k-1) .
\end{array}
$$

The left-hand side of the above equation is a telescopic sum, hence we obtain:

$$
I_{N}=\sum_{k=2}^{N}(-1)^{N-k} \frac{\Gamma(k-1)}{d_{i}^{N-k+1} s^{N-k+1}}-\frac{(-1)^{N}}{d_{i}^{N-1} s^{N-1}} I_{1} .
$$

We calculate $I_{1}$ using integration by parts:

$$
\begin{aligned}
I_{1} & =\int_{0}^{\infty} \frac{e^{-t}}{1+d_{i} s t} d t \\
& =\left.\frac{1}{d_{i} s} \log \left(\frac{1}{d_{i} s+t}\right) e^{-t}\right|_{0} ^{\infty}+\frac{1}{d_{i} s} \int_{0}^{\infty} \log \left(\frac{1}{d_{i} s+t}\right) e^{-t} \\
& \stackrel{\circ}{=}-\frac{\log s+\gamma}{d_{i} s}-\frac{\log d_{i}}{d_{i} s}
\end{aligned}
$$

where in the last step we used the fact $\lim _{\epsilon \rightarrow 0} \int_{0}^{\infty} \log (\epsilon+t) e^{-t}=$ $\Gamma^{\prime}(1)=-\gamma$. Hence we arrive at (19).
Case $1, N \geq M:$ In this case we have:

$$
\left(\prod_{i=1}^{M} d_{i} s\right) \psi_{Z}(-s)=\frac{1}{\Gamma(N)} \int_{0}^{\infty} \frac{t^{N-1} e^{-t}}{\prod_{i=1}^{M}\left(\frac{1}{d_{i} s}+t\right)} d t
$$

we have $\prod_{i=1}^{M}\left(\frac{1}{d_{i} s}+t\right) \geq t^{M}$, now using the dominated convergence theorem we can conclude that:

$$
\begin{aligned}
\left(\prod_{i=1}^{M} d_{i} s\right) \psi_{Z}(-s) & \stackrel{\circ}{=} \frac{1}{\Gamma(N)} \int_{0}^{\infty} t^{N-M-1} e^{-t} d t \\
& =\frac{\Gamma(N-M)}{\Gamma(N)}
\end{aligned}
$$

hence, for $N>M$ we have

$$
\psi_{Z}(-s) \stackrel{\circ}{=} \frac{\Gamma(N-M)}{\Gamma(N) \prod_{i=1}^{M} d_{i}} \frac{1}{s^{M}} .
$$

Case 2 and 3, $N \leq M$

$$
\begin{aligned}
\psi_{Z}(-s) & =\frac{1}{\Gamma(N)} \int_{0}^{\infty} \frac{t^{N-1} e^{-t}}{\prod_{i=1}^{M}\left(1+d_{i} s t\right)} d t \\
& =\frac{1}{\Gamma(N)} \int_{0}^{\infty} \sum_{i=1}^{M} \frac{\alpha_{i} t^{N-1} e^{-t}}{1+d_{i} s t} d t \\
& =\frac{1}{\Gamma(N)} \sum_{i=1}^{M} \alpha_{i} \int_{0}^{\infty} \frac{t^{N-1} e^{-t}}{1+d_{i} s t} d t .
\end{aligned}
$$

From Lemma 3 we obtain

$$
\begin{aligned}
\psi_{Z}(-s) \stackrel{\circ}{=} & \frac{1}{\Gamma(N)} \sum_{i=1}^{N} \alpha_{i}\left(\sum_{k=1}^{N-1} \frac{(-1)^{k-1} \Gamma(N-k)}{d_{i}^{k} s^{k}}\right. \\
& \left.+\frac{(-1)^{N-1}(\log s+\gamma)}{d_{i}^{N} s^{N}}+\frac{(-1)^{N-1} \log d_{i}}{d_{i}^{N} s^{N}}\right)
\end{aligned}
$$

which can be rewritten as

$$
\begin{aligned}
\psi_{Z}(-s) \stackrel{\circ}{=} & \frac{1}{\Gamma(N)} \sum_{k=1}^{N-1}\left(\sum_{i=1}^{M} \frac{\alpha_{i}}{d_{i}^{k}}\right) \frac{(-1)^{k-1} \Gamma(N-k)}{s^{k}} \\
& +\frac{(-1)^{N-1}}{\Gamma(N)} \frac{(\log s+\gamma)}{s^{N}} \sum_{i=1}^{M} \frac{\alpha_{i}}{d_{i}^{k}} \\
& +\frac{(-1)^{N-1}}{\Gamma(N) s^{N}} \sum_{i=1}^{M} \frac{\alpha_{i} \log d_{i}}{d_{i}^{N}} .
\end{aligned}
$$

If $N=M$, by Lemma 2 the inner summation of the term (21a) becomes zero, the summation in term (21b) becomes $\frac{(-1)^{N-1}}{\prod^{N} d_{i}}$, and we get

$$
\begin{aligned}
& \psi_{Z}(-s) \stackrel{\circ}{=} \frac{1}{\Gamma(N) \prod_{i=1}^{N} d_{i}} \frac{\log s+\gamma}{s^{N}} \\
& +\frac{(-1)^{N-1}}{\Gamma(N) s^{N}} \sum_{i=1}^{N} \frac{\alpha_{i} \log d_{i}}{d_{i}^{N}} \\
& \stackrel{\circ}{\Gamma(N) \prod_{i=1}^{N} d_{i}} \frac{\log s}{s^{N}} .
\end{aligned}
$$

If $N<M$, by Lemma 2 the inner summation of the term (21a) and the summation in term (21b) become zero and we obtain:

$$
\psi_{Z}(-s) \stackrel{\circ}{=} \frac{(-1)^{N-1}}{\Gamma(N)}\left(\sum_{i=1}^{M} \frac{\alpha_{i} \log d_{i}}{d_{i}^{N}}\right) \frac{1}{s^{N}} .
$$




\section{REFERENCES}

[1] D. Chizhik, G. J. Foschini, M. J. Gans, and R. A. Valenzuela, "Keyholes, correlations, and capacities of multielement transmit and receive antennas," IEEE Trans. Wireless Commun., vol. 1, no. 2, pp. 361-368, Apr. 2002.

[2] D. Gesbert, H. Bölcskei, D. A. Gore, and A. J. Paulraj, "Outdoor MIMO wireless channels: Models and performance prediction," IEEE Trans. Commun., vol. 50, no. 12, pp. 1926-1934, Dec. 2002.

[3] H. Shin and J. H. Lee, "Effect of keyholes on the symbol error rate of space-time block codes," IEEE Commun. Lett., vol. 7, no. 1, pp. 27-29, Jan. 2003.

[4] A. M. Nasri Nasrabadi, H. R. Bahrami, and S. H. Jamali, "Design of space-time trellis codes for keyhole channels: Performance criterion and code design," IEE Electron. Lett., vol. 40, no. 1, pp. 53-55, Jan. 2004.

[5] S. Sanayei, A. Hedayat, and A. Nosratinia, "Space-time codes in keyhole channels: Analysis and design," in Proc. IEEE Global Telecommun. Conf., Nov.-Dec. 2004, vol. 6, pp. 3768-3772.

[6] M. K. Simon and M.-S. Alouini, Digital Communication over Fading Channels: A Unified Approach to Performance Analysis. New York: John Wiley and Sons, 2000.

[7] V. Tarokh, N. Seshardi, and A. R. Calderbank, "Space-time codes for high data rate wireless communication: Performance criteria and code construction," IEEE Trans. Inform. Theory, vol. 44, no. 2, pp. 744-765, Mar. 1998.

[8] E. Malkamäki and H. Leib, "Evaluating the performance of convolutional codes over block fading channels," IEEE Trans. Inform. Theory, vol. 45, no. 5, pp. 1643-1646, July 1999.

[9] H. Jafarkhani and N. Seshadri, "Super-orthogonal space-time trellis codes," IEEE Trans. Inform. Theory, vol. 49, no. 4, pp. 937-950, Apr. 2003. 